\title{
Key performance indicators - a tool to answer the questions, what do we get for our money? and how can we use this money even more efficiently?
}

\author{
M Bork Hansen
}

From 26th Patient Classification Systems International (PCSI) Working Conference
Munich, Germany. 15-18 September 2010

\section{Introduction}

Introducing key performance indicators is part of a government strategy, and a first step to an improved overview on the use of resources in the Danish hospitalcare sector. It also answers the question, what do we get for our money?

Publishing these key performance indicators will make best practice visible, and thereby provide information on how to use resources even more efficiently. To give hospitals an incentive to act in the direction of best practice, payment for results that create more value and quality for money should be used as a supplement to the published key performance indicators.

\section{Methods}

Based on data from the National Patient Registry, the indicators are calculated on a national, regional and hospital level.

Also, relevant indicators are calculated for all 98 municipalities since they share the responsibility along with the hospitals (if necessary) to take patients home after a hospitalization.

The value of each specific DRG case-mix group reflects the cost of an average patient within the specific group. To reflect the cost of an average patient across the least expensive, most cost-efficient hospitals, a bestpractice value is calculated for each of the DRG casemix groups based on data from the Danish Patient Cost Database.

\section{Results}

The idea is to improve transparency, provide a quick management overview and, by linking activity and costs, make it possible for regions, hospitals and municipalities to use both key performance indicators and best-practice values to benchmark and, thereby, impact performance in the direction of best practice.

\section{Conclusions}

This is a first step towards introducing quality measures in the DRG payment of hospitals and turning the incentive structure in the direction of payment for results that create more value and/or health for money.

The vision is to connect case mix - information, clinical outcomes and payment.

Published: 6 October 2010

doi:10.1186/1472-6963-10-S2-A11

Cite this article as: Hansen: Key performance indicators - a tool to answer the questions, what do we get for our money? and how can we use this money even more efficiently?. BMC Health Services Research 2010 10(Suppl 2):A11. 